\title{
Sorghum and wheat differentially affect caecal microbiota and associated performance characteristics of meat chickens
}

\author{
Eduardo Crisol-Martínez ${ }^{\text {Corresp., }}{ }^{1}$ ， Dragana Stanley ${ }^{1,2,3}$ ， Mark S Geier ${ }^{4}$ ， Robert J Hughes ${ }^{3,5,6}$ ， Robert J Moore ${ }^{3,}$ \\ 7,8 \\ ${ }^{1}$ School of Medical and Applied Sciences, Central Queensland University, Rockhampton, Queensland, Australia \\ 2 Institute for Future Farming Systems, Central Queensland University, Rockhampton, Queensland, Australia \\ 3 Poultry Cooperative Research Centre, University of New England, Armidale, New South Wales, Australia \\ 4 Research and Innovation Services, University of South Australia, Mawson Lakes, South Australia, Australia \\ 5 Pig and Poultry Production Institute, South Australian Research and Development Institute, Roseworthy, South Australia, Australia \\ ${ }^{6}$ School of Animal and Veterinary Sciences, The University of Adelaide, Roseworthy, South Australia, Australia \\ 7 School of Science, RMIT University, Bundoora, Victoria, Australia \\ 8 Department of Microbiology, Monash University, Clayton, Victoria, Australia \\ Corresponding Author: Eduardo Crisol-Martínez \\ Email address: eduardocrisol@gmail.com
}

This study compared the effects of wheat- and sorghum-based diets on broiler chickens. The growth performance and caecal microbial community of chickens were measured and correlations between productivity and specific gut microbes were observed. Cobb broilers 15 days of age were individually caged and two dietary treatments were used, one with a wheat-based diet $(n=48)$ and another one with a sorghum-based diet $(n=48)$. Growth performance measurements were taken over a 10 day period and samples for microbiota analysis were taken at the end of that period. Caecal microbiota was characterised by sequencing of $16 \mathrm{~S}$ bacterial rRNA gene amplicons. Overall, the results indicated that a sorghum-based diet produced higher apparent metabolisable energy (AME) and bodyweight gain (BWG) values in chickens, compared to a wheat-based diet. Nevertheless, sorghum-fed birds had higher feed conversion ratio (FCR) values than wheat-fed birds, possibly because of some anti-nutritional factors in sorghum. Further analyses showed that caecal microbial community was significantly associated with AME values, but microbiota composition differed between dietary treatments. A number of bacteria were individually correlated with growth performance measurements. Numerous OTUs assigned to strains of Lactobacillus crispatus and Lachnospiraceae, which were prevalent in sorghum-fed chickens, were correlated with high AME and BWG values, respectively. Additionally, a number of OTUs assigned to Clostridiales that were prevalent in wheat-fed chickens, were correlated with low FCR values. Overall, these results suggest that between-diet variations in growth performance were partly associated with changes in the caecal microbiota. 


\section{Sorghum and wheat differentially affect caecal microbiota}

\section{2 and associated performance characteristics of meat chickens}

3 Eduardo Crisol-Martínez ${ }^{1, *}$, Dragana Stanley ${ }^{1,2,3,{ }^{*}, \text { Mark S. Geier }}{ }^{4}$, Robert J. Hughes ${ }^{3,5,6}$, Robert

4 J. Moore $3,7,8$

$5{ }^{1}$ School of Medical and Applied Sciences, Central Queensland University, Rockhampton,

6 Queensland, Australia

$7 \quad 2$ Institute for Future Farming Systems, Central Queensland University, Rockhampton,

8 Queensland, Australia

$9{ }^{3}$ Poultry Cooperative Research Centre, University of New England, Armidale, New South

10 Wales, Australia

$11{ }^{4}$ Research and Innovation Services, University of South Australia, Mawson Lakes, South

12 Australia, Australia.

$13{ }^{5}$ Pig and Poultry Production Institute, South Australian Research and Development Institute,

14 Roseworthy, South Australia, Australia

$15{ }^{6}$ School of Animal and Veterinary Sciences, The University of Adelaide, Roseworthy, South

16 Australia, Australia

$17{ }^{7}$ School of Science, RMIT University, Bundoora, Victoria, Australia

$18{ }^{8}$ Department of Microbiology, Monash University, Clayton, Victoria, Australia

$19{ }^{*}$ These two authors contributed equally to this work

20 Corresponding author: Eduardo Crisol-Martínez

21 Current address: Central Queensland University, 120 Spencer Street, Melbourne, Victoria, 3000,

22 Australia

23 Email: eduardocrisol@gmail.com 


\section{Abstract}

25 This study compared the effects of wheat- and sorghum-based diets on broiler chickens. The growth performance and caecal microbial community of chickens were measured and correlations between productivity and specific gut microbes were observed. Cobb broilers 15 days of age were individually caged and two dietary treatments were used, one with a wheatbased diet $(\mathrm{n}=48)$ and another one with a sorghum-based diet $(\mathrm{n}=48)$. Growth performance measurements were taken over a 10 day period and samples for microbiota analysis were taken at the end of that period. Caecal microbiota was characterised by sequencing of $16 \mathrm{~S}$ bacterial rRNA gene amplicons. Overall, the results indicated that a sorghum-based diet produced higher apparent metabolisable energy (AME) and body-weight gain (BWG) values in chickens, compared to a wheat-based diet. Nevertheless, sorghum-fed birds had higher feed conversion ratio (FCR) values than wheat-fed birds, possibly because of some anti-nutritional factors in sorghum. Further analyses showed that caecal microbial community was significantly associated with AME values, but microbiota composition differed between dietary treatments. A number of bacteria were individually correlated with growth performance measurements. Numerous OTUs assigned to strains of Lactobacillus crispatus and Lachnospiraceae, which were prevalent in sorghum-fed chickens, were correlated with high AME and BWG values, respectively. Additionally, a number of OTUs assigned to Clostridiales that were prevalent in wheat-fed chickens, were correlated with low FCR values. Overall, these results suggest that between-diet variations in growth performance were partly associated with changes in the caecal microbiota. 


\section{Introduction}

45

46

47

Wheat, maize and sorghum are the three most commonly used grains in the poultry industry worldwide (Liu et al. 2014). These cereal grains usually constitute a major proportion (60-70\%) of the diet fed to broilers (Black et al. 2005). Although cereals represent a major source of energy for birds, there are wide variations in the energy and nutrient content of different species and cultivars of cereals (Choct \& Hughes 1999). Specifically, wheat is perceived as having a high feeding value for poultry, yet, in reality, some studies have indicated that there are extreme variations in the apparent metabolisable energy (AME) values of wheat-based diets in chickens (Choct et al. [1999] and references within). Sorghum, the other major cereal, presents a number of economic advantages over wheat, since it can be grown under drier conditions, and is usually less costly (Liu et al. 2013). Also, in comparison to wheat, sorghum is thought to have a more consistent, higher energy density (Selle et al. 2010).

It is recognised that diet is the major determinant of the composition of the gastro-intestinal microbiota (Shakouri et al. 2009). A number of components in the diet, such as content of proteins, fats and carbohydrates, can influence the composition of the gut microbiota (Hübener et al. 2002; Rehman et al. 2007). There is a symbiotic relationship between gut microbiota and the host. The complex community of microorganisms, dominated by bacteria, regulates processes related to host metabolism and immunity (Round \& Mazmanian 2009; Tremaroli \& Bäckhed 2012). Thus, it is of interest to the poultry industry to understand the influence of the gut microbiota on the host's nutrient and energy use efficiency. For instance, Stanley et al. (2013) found an association between caecal microbiota composition and productivity in chickens, showing that the abundance of a number of caecal bacteria were correlated with high growth performance values. Thus, a better understanding of the beneficial or detrimental role of intestinal bacteria and how to influence their abundance could result in improvements in broiler performance.

Few studies have analysed the impact of different diets on the structure of the gut microbial community. Additionally, there is limited information on the influence of cereal-based diets on specific microbial populations that could be associated with the health and productivity of the birds (Shakouri et al. 2009). For instance, Torok et al. (2011) found a number of OTUs within the caecum and the ileum which were negatively or positively correlated with feed conversion 
ratio (FCR) values. Lunedo et al. (2014) found increased enterobacteria abundance in the ileum, consistent with the low feed conversion performance of sorghum-fed chickens. In a recent study, our group identified a number of caecal bacteria associated with desirable productivity outcomes on chickens fed with the same diet, and suggested that further investigation could lead to their application as probiotics to improve bird performance (Stanley et al. 2016). In this study, we aimed to expand this information by analysing changes in broiler performance and caecal bacteria across wheat- or sorghum-based diets, and identifying which specific bacteria were associated with parameters of productivity in each dietary treatment.

\section{Materials and methods}

\section{Animal ethics}

The Animal Ethics Committees of the University of Adelaide (Approval No.S-2011-218) and the Department of Primary Industries and Resources, South Australia (Approval No. 25/11) approved this study. All animal work was conducted in accordance with the national and international guidelines for animal welfare.

\section{Animal trials}

Newly-hatched male Cobb 500 broiler chickens from the Baiada Hatchery, Willaston, SA, Australia, were transferred to two floor pens (one for each experimental diet) with wood sawdust and shavings bedding material in an environmentally controlled experimental animal facility. Feed and water were supplied ad libitum throughout the experiment. Two commercially prepared starter diets were used in this study. One was based on wheat $(500 \mathrm{~g} / \mathrm{kg})$ as the main cereal component. The other diet was formulated to the same nutrient specifications but contained sorghum $(300 \mathrm{~g} / \mathrm{k})$ and wheat $(300 \mathrm{~g} / \mathrm{kg})$. Each diet provided $230 \mathrm{~g} / \mathrm{kg}$ crude protein and 12.55 $\mathrm{MJ} / \mathrm{kg}$ as fed, and both contained monensin (65 ppm), nicarbazin (45 ppm) and zinc bacitracin (75 ppm). Both diets also contained commercial exogenous phytase and xylanase enzyme products. The diets were fed from day of hatch (day 0) until the end of the experiment on day 25.

On day 13 , chicks ( $n=48$ for each dietary treatment) were transferred in pairs to 48 metabolism cages in a temperature controlled room $\left(23-25^{\circ} \mathrm{C}\right)$. Initial placing in metabolic cages in pairs was done to minimise stress and allow the birds to adjust to cages. At day 15, birds were moved into individual cages. Individual caging allowed the precise assessment of individual feed intake, 
103

104

105

106

107

108

109

110

111

112

113

114

115

116

117

118

119

120

121

122

123

124

125

126

127

128

129

130

131

132

energy in feed, and unused energy remaining in faeces. The experimental design eliminated competition for feed and reduced behavioural issues affecting feed intake. Single bird caging and individual measurements and sampling were implemented in order to allow direct correlation of microbiota structure and productivity measurements on a bird by bird basis. Birds were euthanised and necropsied on day 25 and caecal contents were collected from each bird and immediately transferred to a $-20^{\circ} \mathrm{C}$ freezer. Samples from all birds were analysed.

FCR was calculated as a ratio of feed eaten and weight gained. Thus birds with low FCR, that needed less feed per kg gained, were the most efficient in converting feed to mass. Gross energy (GE) was measured in feed and in faeces of each individual bird using a Parr isoperibol bomb calorimeter (Parr Instrument Company, Moline, IL). Apparent metabolisable energy (AME) in $\mathrm{MJ} / \mathrm{kg}$ dry matter, was calculated as $\left(\mathrm{AME}_{\text {diet }}=\left[\left(\mathrm{GE}_{\text {diet }} \mathrm{x}\right.\right.\right.$ feed eaten $) \mathrm{x}\left(\mathrm{GE}_{\text {excreta }} \mathrm{x}\right.$ dry excreta)]/feed eaten/dry diet content). Body weight gain (BWG) was calculated as [weight gain (g) / start weight (g)] and feed eaten (FE) was total amount of feed eaten during the 10 day measurement time period. All of the above measurements were taken from day 15 to day 25 , during the time when single birds were housed in metabolic cages.

\section{DNA preparation, PCR amplification of $16 S \mathrm{rRNA}$ gene sequences, and bioinformatic}

\section{analysis}

DNA was isolated using the method of Yu \& Morrison (2004), briefly, raw DNA extracts were obtained by initial lysis of caecal content in lysis buffer at $70{ }^{\circ} \mathrm{C}$, followed by bead beating using a Precellys 24 instrument (Stretton Scientific Limited). This was followed by enzyme removal of RNA and protein and subsequent purification using Qiagen columns as per the supplier's instruction. TheV1-V3 region of the 16S rRNA gene was amplified (forward primer [Lane 1991], 5' AGAGTTTGATCCTGG 3'; reverse primer W31 [Snell-Castro et al. 2005], 5' TTACCGCGGCTGCT 3') as detailed by Stanley et al. (2012). Pyrosequencing was performed using a Roche/454 FLX+ instrument and Titanium chemistry kits according to the manufacturer's instructions. Sff file processing was done using PyroBayes (Quinlan et al. 2008) and inspected for chimeric sequences using Pintail (Ashelford et al. 2005) and error corrected using Acacia (Bragg et al. 2012). Further trimming was done in QIIME (Caporaso et al. 2010) with sequence length 300-600 bases, no ambiguous sequences, minimum average quality score of 25 and maximum of 6 bases in homopolymer runs. OTU picking was done using Uclust 
133 (Edgar 2010). Taxonomy was assigned using Blast against the GreenGenes database (DeSantis et 134 al. 2006) and QIIME v.1.8 defaults. Additional taxonomic assignments for OTUs of interest was 135 performed using EzTaxon database (Chun et al. 2007). The complete dataset is available on MG136 RAST database (http://metagenomics.anl.gov/) under library ID mg1538406.

\section{Statistical analysis}

138 Permutational analysis of variance (PERMANOVA) (Anderson 2001) was used to analyse 139 differences between dietary treatments on each of the four performance measures (AME, FCR, $140 \mathrm{BWG}$ and FE) and also for differences in microbiota composition. The distance matrices were 141 based on Euclidean distance (growth performance data) and both Weighted and Unweighted 142 Unifrac distances (microbiota abundance data) (Lozupone \& Knight 2005). The PERMDISP 143 routine was used to test for the homogeneity of dispersions (based on mean distance to group 144 centroids), to ensure that dispersions were constant among groups (Anderson 2006). Principal 145 Coordinates Analysis (PCO) was used to visualise between-groups differences in microbiota 146 composition, based on both Unifrac distances. Analysis of variance (ANOVA) was used to test

147 for differences in the diversity of microbial community between dietary treatments using four 148 diversity measures (i.e. evenness, richness, Shannon's and Inverted Simpson's indices). ANOVA 149 was also used to test for differences in the abundance of individual microbial taxa between 150 dietary treatments. Redundancy discrimination analysis (RDA) was used to analyse individual associations between microbiota community composition and each of the performance measures

152 in both dietary treatments. Pearson's correlation tests were conducted to analyse the correlations 153 between performance indicators. Spearman's correlation test was used to analyse correlations 154 between individual OTUs and each of the performance indicators. For all ANOVA, RDA and 155 correlation analyses, microbiota abundance data was normalised and square root transformed to 156 reduce variance heterogeneity and increase predictive power. PERMANOVA, PERMDISP and 157 PCO analyses were carried out using PRIMER software (v.6.1.16) with the PERMANOVA+ 158 add-on (v.1.0.6). RDA, Pearson's and Spearman's correlation tests, and analyses of community 159 diversity and taxonomic structure were performed in Calypso (v.5.8)

160 (http://cgenome.net/calypso/faces/uploadFiles.jsp).

\section{Results}

\section{Growth performance measures}


163 Dietary treatment caused significant differences in all four performance measures (Fig. 1).

164 Chickens fed with sorghum had significantly higher FCR values ( $1.50 \pm 0.01$ [average \pm standard

165

166

167

168

169

170

171

172

173

174

175

176

177

178 error]) than those fed with wheat $(1.47 \pm 0.01)(\mathrm{P}=0.004)$ (Fig. 1A). Additionally, AME values were significantly higher in sorghum-fed chickens $(14.71 \pm 0.04)$ than in wheat-fed birds $(14.44$ $\pm 0.06)(\mathrm{P}=0.002)$ (Fig. 1B). BWG values were also significantly higher in birds fed with sorghum $(886 \pm 12)$ than in those fed with wheat $(843 \pm 10)(\mathrm{P}=0.004)$ (Fig. 1C). Lastly, chickens had significantly higher FE values when fed with sorghum $(1331 \pm 17)$ than when fed with wheat $(1240 \pm 16)(\mathrm{P}=0.002)$ (Fig. 1D). PERMDISP results showed that none of the latter PERMANOVA results were significantly affected by data dispersion (all with $\mathrm{P}>0.05$ ).


Fig. 1 Boxplots showing differences in four growth performance measures between diet treatments. 'Sorghum' refers to birds fed with a sorghum-based diet, whereas 'wheat' refers to those fed with a wheat-based diet. Significant differences between treatments are indicated ('**’: $\mathrm{P} \leq 0.01$ ).

Pearson's correlation test results indicated that in each dietary treatment, AME was significantly negatively correlated with BWG (wheat: $\mathrm{r}=-0.382, \mathrm{P}=0.010$; sorghum: $\mathrm{r}=-0.410, \mathrm{P}=0.005$ ) and FE (wheat: $\mathrm{r}=-0.408, \mathrm{P}=0.005$; sorghum: $\mathrm{r}=-0.506, \mathrm{P} \leq 0.001$ ). However, FCR and FE were 
179 significantly positively correlated in wheat-fed chickens $(\mathrm{r}=0.311, \mathrm{P}=0.037)$, but not in sorghum180 fed chickens $(\mathrm{r}=0.155, \mathrm{P}=0.308)$.

181

182

\section{Structure of the caecal microbial community}

The microbiota profile comparison at the family level indicated high similarities between dietary treatments, both dominated by Lactobacillaceae, Lachnospiraceae and Ruminococcaceae (Fig. 2).



Fig. 2 Caecal microbiota profiles showing normalised square root transformed abundances of the families in sorghum-fed chickens (A) and wheat-fed chickens (B).

None of the four selected diversity measures (i.e. richness, evenness, Shannon's and inverted Simpson's indices) varied significantly between dietary treatments, neither at the OTU level, nor at any higher taxonomic level $(\mathrm{P}>0.05)$. Comparisons of the microbiota composition at the OTU level revealed significant differences between treatments when Unweighted Unifrac Distances were used (Pseudo-F=2.65, $\mathrm{P}=0.006$ ) (Fig. 3A), but not when Weighted Unifrac Distances were used (Pseudo-F=2.59, $\mathrm{P}=0.063$ ) (Fig. 3B). PERMDISP confirmed that the significant result 
194 observed using Unweighted Unifrac Distances did not occur due to differences in data dispersion $195(\mathrm{P}=0.640)$.
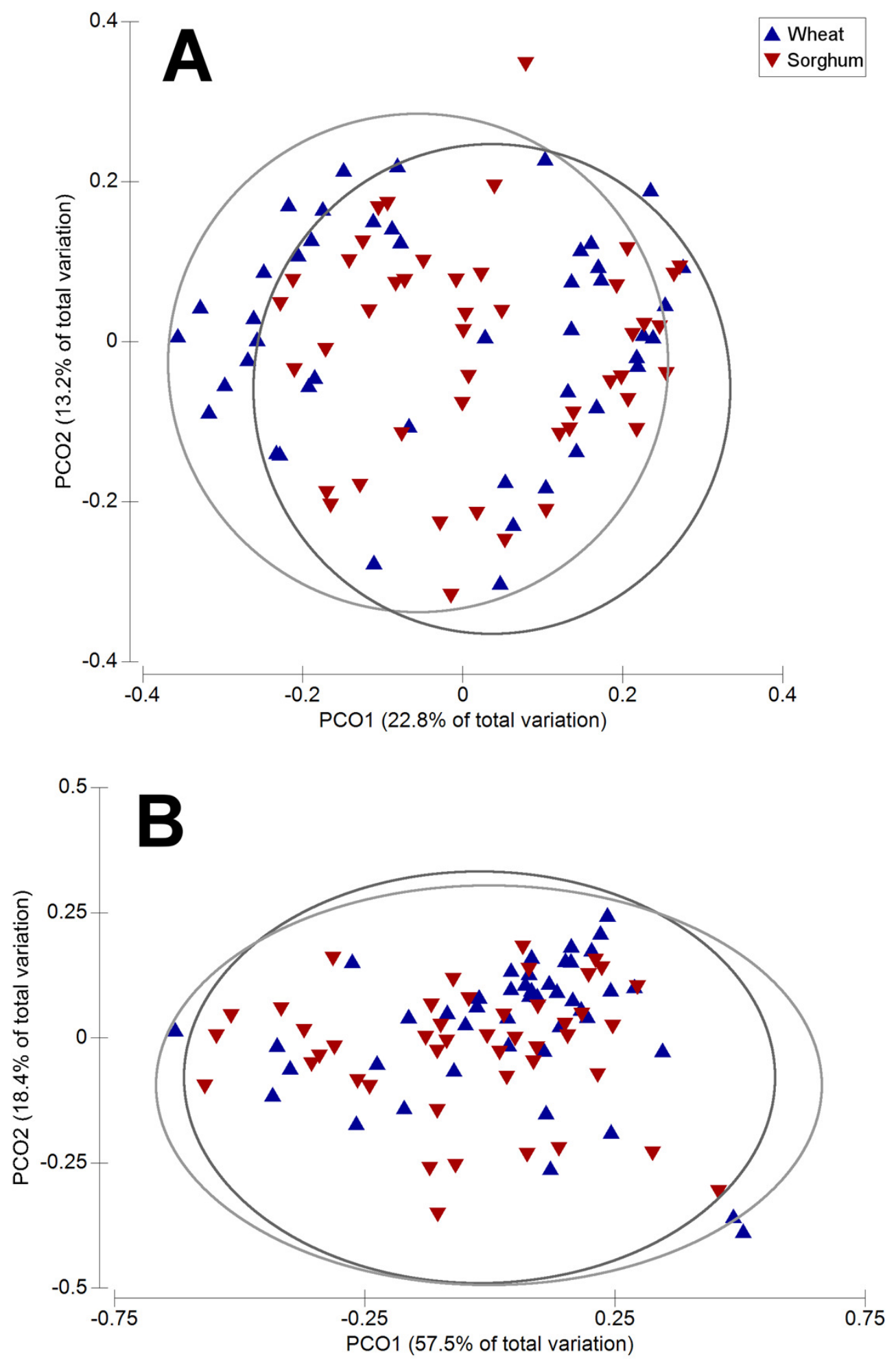
197 Fig. 3 Principal Coordinates Analysis (PCO) showing a two-dimensional ordination of gut microbiota composition

198 at the OTU level by dietary treatment (indicated by symbols). 'A' plot is based on Unweighted Unifrac distance,

199 whereas 'B' plot is based on Weighted Unifrac distance. Overlayed grey ellipses in were created to help visualise

200 the between-treatment differences in composition. Axes indicate the percentage of variation in the data.

\section{Gut microbiota shifts induced by diet}

202 Diet differences produced significant changes $(\mathrm{P} \leq 0.05)$ in 98 out of 522 OTUs $(18.8 \%)$. At the

203

204

205

206

207

208

209

210

211

212

213

214

215

216

217

218 species level, sorghum-fed birds showed significantly higher abundances of a phylotype closest to Lactobacillus crispatus (identity assigned to 21 OTUs ( $\mathrm{n}=21$ ), all DSM 20584(T) strains, with an averaged similarity (avg. sim.) of 92.67\% [EzTaxon]) than wheat-fed birds ( $\mathrm{P} \leq 0.001)$ (Fig. $4 \mathrm{~A}$ ). Out of the 32 OTUs allocated to this species, OTU3 (with a similarity of $100 \%$ ) was the most abundant and showed a similar trend as seen for the whole L. crispatus phylotype ( $\mathrm{P} \leq 0.001$ ) (Fig. 4B). Showing an opposite trend, the numbers of a phylotypes most similar to Clostridium leptum ( $\mathrm{n}=30$, all DSM753 ( $\mathrm{T}$ ), avg. sim. $=87.94 \%$ ) were significantly higher in chickens fed with wheat than in those fed with sorghum ( $\mathrm{P}=0.022)$ (Fig. 4A). At the genus level, wheat-fed birds showed significant lower abundances of Enterococcus and Coprococcus than sorghum-fed birds ( $\mathrm{P}=0.016$ and $\mathrm{P}=0.018$, respectively) (Fig. $4 \mathrm{C})$. Shifts in the Enterococcus genus (Fig. 4C) translated into equal changes at the family level (Enterococcaceae) (Fig. 4D). Also, the Lachnospiraceae family had significantly higher numbers in chickens fed with sorghum than in those fed with wheat $(\mathrm{P}=0.003)$. Additionally, two families, Clostridiales family XIII and Catabacteriaceae, showed significantly higher abundances in wheat-fed chickens than in sorghum-fed chickens ( $\mathrm{P}=0.008$ and $\mathrm{P}=0.012$, respectively) (Fig. 4D). No other significant shifts were found at any other taxonomic level $(\mathrm{P}>0.05)$. 


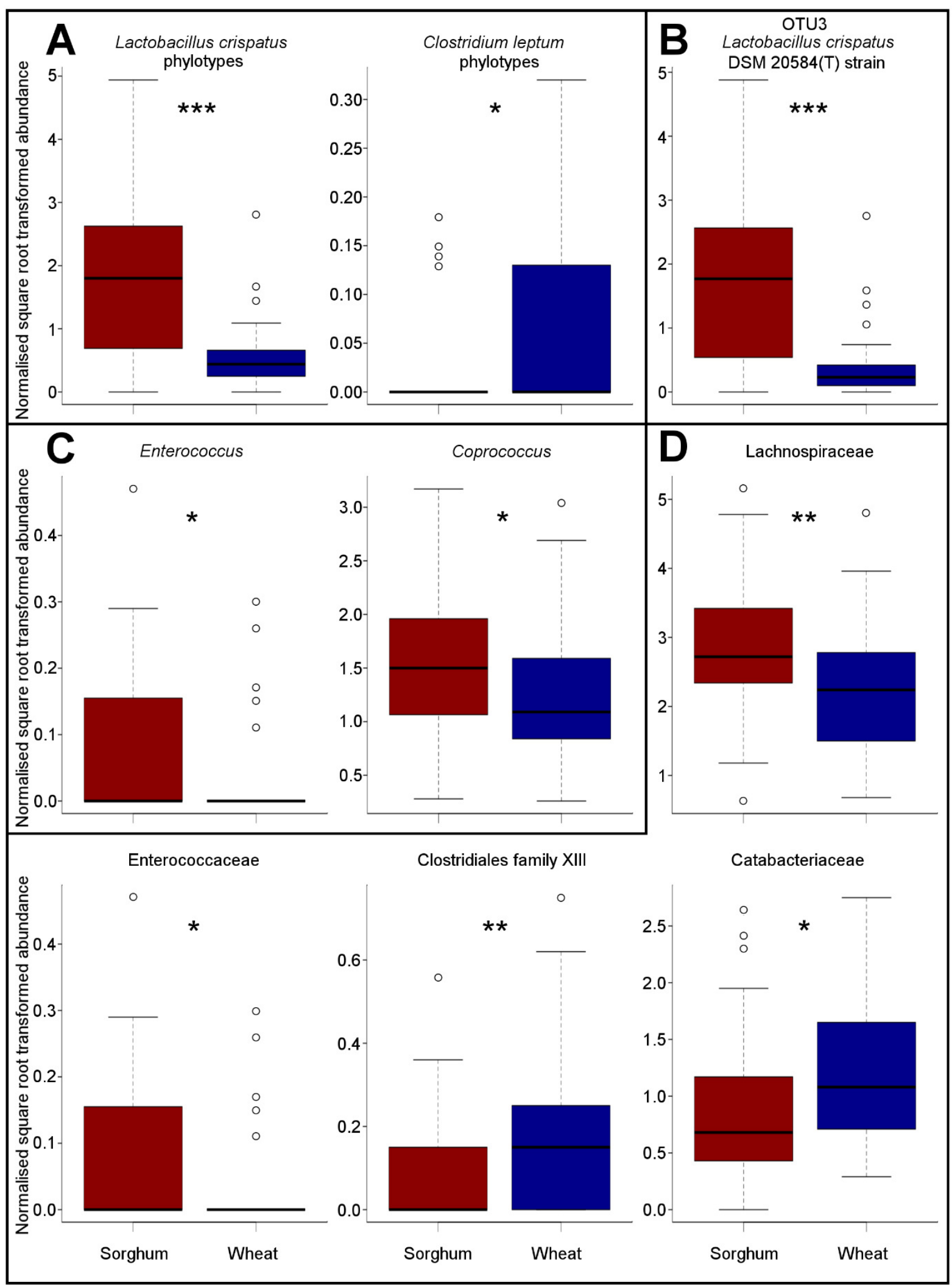


220

221

222

223

224

225

226

227

228

229

230

231

232

233

234

235

236

237

238

239

240

241

242

243

244

245

246

247

248

249

Fig. 4 Caecal microbiota showing significant differences in normalised square root transformed abundance between dietary treatments (indicated by colours). 'A', 'C' and 'D' show taxa at, respectively, the species, genus and family levels. 'B' shows the shift in OTU3, which identity was assigned (using EzTaxon) to 100\% L. crispatus DSM 20584(T) strain. Statistically significant differences are indicated ('*’: $\mathrm{P} \leq 0.05$; ‘**’: $\mathrm{P} \leq 0.01$; ‘***’: $\mathrm{P} \leq 0.001) . L$. crispatus phylotype was assigned to 21 OTUs, all DSM 20584(T) strains, with an averaged similarity (avg. sim.) of 92.67\%. Clostridium leptum phylotype was assigned to 30 OTUs, all DSM 753(T) strains (avg. sim.=87.94\%).

\section{Associations between microbiota and performance measures}

The RDA analysis showed that, at the OTU level in both dietary treatments, gut microbiota composition was significantly associated with $\operatorname{AME}(\mathrm{P}=0.007)$, but not to FCR $(\mathrm{P}=0.206), \mathrm{BWG}$ $(\mathrm{P}=0.156)$ or $\mathrm{FE}(\mathrm{P}=0.061)$. Out of the 98 OTUs showing significant shifts between dietary treatments, 54 OTUs (53.02\%) showed individual, significant correlations with at least one of the growth performance measures (table 1). More than $50 \%$ of these OTUs $(n=30)$ were significantly correlated with AME values, and all the OTUs showing positive correlations $(n=11)$ were significantly more abundant in sorghum-fed birds than in wheat-fed birds (Table 1). Particularly, four OTUs assigned to a phylotype closest to L. crispatus (OTUs 3, 221, 35 and 503) showed consistent positive correlations with AME values. Indeed, L. crispatus phylotypes were positively correlated with $\operatorname{AME}(\rho=0.268, \mathrm{P}=0.011)$. On the contrary, all the OTUs showing negative correlations with AME $(n=19)$ were significantly more abundant in wheat-fed chickens. Most of these OTUs were assigned to either the genus Clostridium or the order Clostridiales (OTUs 401, 664, 299, 429, 127, 340, 244, 92, 261, 163, 16, 199, 342 and 148). Nevertheless, some other OTUs within the order Clostridiales were positively correlated with growth performance: nine OTUs assigned to this order showed significant, negative correlations with FCR values (OTUs 401, 127, 92, 261, 409, 550, 355, 682 and 416). Moreover, the family Clostridiaceae was positively correlated with $\operatorname{AME}$ values $(\rho=0.250, \mathrm{P}=0.017)$. Additionally, eight OTUs (assigned to various taxa) which were significantly prevalent in sorghum-fed birds, showed a significant positive correlation with BWG values (OTUs 123, 511, 162, 43, 54, 192 , 321 and 9). At higher taxonomic levels, Lactobacillus helveticus was significantly negatively correlated with BWG $(\rho=-0.210, \mathrm{P}=0.047)$. No other taxa at any other taxonomic level showed significant correlations with any of the growth performance measures. 
250 Table 1. Correlation values based on Spearman's tests between OTUs showing significant shifts between dietary

251 treatments and growth performance measures. $\rho$ values were ordered from largest to smallest. The significant level

252 of each correlation is indicated. Colours indicate whether a given OTU showed significantly higher mean abundance

253 (normalised square root transformed values) in sorghum-fed birds (red) or wheat-fed birds (blue), and the significant

254 level is indicated as ' $\mathrm{P}$ (diet)'. Taxonomic assignment is based on the furthermost default QIIME taxonomy against

255 GreenGenes database. ('ns': not significant; '*': $\mathrm{P} \leq 0.05$; ‘**': $\mathrm{P} \leq 0.01$; ‘***': $\mathrm{P} \leq 0.001$ ).

\begin{tabular}{|c|c|c|c|c|c|c|c|c|}
\hline \multirow{2}{*}{$\begin{array}{c}\text { OTU } \\
\text { no. }\end{array}$} & \multirow[b]{2}{*}{ Taxon } & \multirow{2}{*}{$\begin{array}{c}\mathrm{P} \\
\text { (diet) }\end{array}$} & \multirow[b]{2}{*}{ Sorghum } & \multirow[b]{2}{*}{ Wheat } & \multicolumn{4}{|c|}{$\rho$ values } \\
\hline & & & & & AME & FCR & $\mathrm{BWG}$ & $\mathrm{FE}$ \\
\hline 3 & Lactobacillus crispatus & $* * *$ & 1.79 & 0.37 & $0.337 * *$ & ns & ns & ns \\
\hline 221 & Lactobacillus crispatus & $* *$ & 0.11 & 0.02 & $0.332 * *$ & ns & ns & ns \\
\hline 305 & Clostridium & $* * *$ & 0.13 & 0.03 & $0.331 * *$ & ns & ns & ns \\
\hline 35 & Lactobacillus crispatus & $* *$ & 0.03 & 0.00 & $0.290 * *$ & ns & ns & ns \\
\hline 66 & Clostridium & $* * *$ & 0.38 & 0.11 & $0.261^{*}$ & ns & ns & ns \\
\hline 714 & Lactobacillus & $* *$ & 0.23 & 0.05 & $0.251 *$ & ns & ns & ns \\
\hline 318 & Clostridiales & $* * *$ & 0.10 & 0.01 & $0.235^{*}$ & ns & ns & ns \\
\hline 247 & Ruminococcus & $* * *$ & 0.26 & 0.07 & $0.230 *$ & ns & ns & ns \\
\hline 112 & Faecalibacterium & $* * *$ & 0.19 & 0.03 & $0.219 *$ & ns & ns & ns \\
\hline 503 & Lactobacillus crispatus & $*$ & 0.09 & 0.05 & $0.209 *$ & ns & ns & ns \\
\hline 8 & Clostridiales & $* *$ & 1.29 & 0.48 & $0.207^{*}$ & ns & ns & ns \\
\hline 401 & Clostridiales Family & $* *$ & 0.06 & 0.14 & $-0.208^{*}$ & $-0.233^{*}$ & ns & ns \\
\hline 664 & Clostridiales & $*$ & 0.02 & 0.11 & $-0.210^{*}$ & ns & ns & ns \\
\hline 299 & Clostridiales & $*$ & 0.00 & 0.02 & $-0.211^{*}$ & ns & ns & ns \\
\hline 245 & Ruminococcaceae & $*$ & 0.02 & 0.07 & $-0.212 *$ & ns & ns & ns \\
\hline 429 & Clostridiales & $*$ & 0.02 & 0.12 & $-0.213^{*}$ & ns & ns & ns \\
\hline 127 & Clostridiales & $* * *$ & 0.01 & 0.09 & $-0.216^{*}$ & $-0.283 * *$ & ns & ns \\
\hline 340 & Clostridiales & $*$ & 0.00 & 0.04 & $-0.217^{*}$ & ns & ns & ns \\
\hline 244 & Clostridiales & $*$ & 0.01 & 0.05 & $-0.226^{*}$ & ns & ns & ns \\
\hline 92 & Clostridiales & $*$ & 0.12 & 0.26 & $-0.231 *$ & $-0.222 *$ & ns & ns \\
\hline 261 & Clostridiales & $* * *$ & 0.15 & 0.69 & $-0.233^{*}$ & $-0.229 *$ & ns & ns \\
\hline 163 & Clostridiales & $* *$ & 0.27 & 0.66 & $-0.235^{*}$ & ns & ns & ns \\
\hline 16 & Clostridiales & $*$ & 0.05 & 0.22 & $-0.239 *$ & ns & ns & ns \\
\hline 199 & Clostridiales & $*$ & 0.03 & 0.20 & $-0.247 *$ & ns & ns & ns \\
\hline 202 & Coprococcus & $* * *$ & 0.05 & 0.22 & $-0.257^{*}$ & $-0.242 *$ & ns & ns \\
\hline 589 & Ruminococcaceae & $*$ & 0.00 & 0.02 & $-0.265^{*}$ & $-0.211 *$ & $\mathrm{~ns}$ & $\mathrm{~ns}$ \\
\hline 77 & Lachnospiraceae & $* * *$ & 0.16 & 0.42 & $-0.268^{*}$ & ns & $\mathrm{ns}$ & ns \\
\hline 21 & Faecalibacterium & $* *$ & 0.67 & 1.16 & - & ns & ns & $\mathrm{ns}$ \\
\hline 342 & Clostridiales & $*$ & 0.00 & 0.03 & - & ns & ns & ns \\
\hline 148 & Clostridiales & $* *$ & 0.03 & 0.13 & - & ns & ns & ns \\
\hline 123 & Clostridium & $* * *$ & 0.17 & 0.02 & ns & $0.378 * * *$ & $0.251 *$ & $0.384 * * *$ \\
\hline 409 & Clostridiales & $* *$ & 0.00 & 0.04 & ns & $-0.208^{*}$ & ns & $\mathrm{ns}$ \\
\hline 550 & Clostridiales & $*$ & 0.00 & 0.03 & ns & $-0.219^{*}$ & ns & ns \\
\hline 48 & Catabacteriaceae & $* * *$ & 0.18 & 0.54 & ns & $-0.219^{*}$ & ns & ns \\
\hline 355 & Clostridiales & $*$ & 0.19 & 0.52 & ns & $-0.224^{*}$ & ns & ns \\
\hline 682 & Clostridiales & $*$ & 0.00 & 0.03 & ns & $-0.228^{*}$ & ns & ns \\
\hline 68 & Tenericutes order & $* * *$ & 0.07 & 0.31 & $\mathrm{~ns}$ & $-0.236^{*}$ & ns & ns \\
\hline 416 & Clostridiales & $* *$ & 0.00 & 0.14 & ns & $-0.242 *$ & ns & ns \\
\hline 266 & Ruminococcaceae & $*$ & 0.01 & 0.06 & ns & $-0.290 * *$ & ns & ns \\
\hline 85 & Ruminococcus & $* * *$ & 0.03 & 0.17 & ns & $-0.308 * *$ & ns & ns \\
\hline 233 & Blautia & $*$ & 0.04 & 0.09 & ns & $-0.341 * *$ & ns & $-0.218^{*}$ \\
\hline 511 & Clostridiales & $*$ & 1.78 & 1.49 & ns & ns & $0.301 * *$ & $0.277 * *$ \\
\hline 162 & Lactobacillus & $*$ & 0.17 & 0.11 & ns & $\mathrm{ns}$ & $0.284 * *$ & $0.329 * *$ \\
\hline 43 & Coprococcus & $* *$ & 0.68 & 0.47 & ns & $\mathrm{ns}$ & $0.279 * *$ & $0.237^{*}$ \\
\hline 54 & Lachnospiraceae & $*$ & 0.59 & 0.44 & ns & ns & $0.234^{*}$ & $0.253^{*}$ \\
\hline
\end{tabular}




\begin{tabular}{clcccccccc}
192 & Lactobacillus & $*$ & 0.22 & 0.10 & $\mathrm{~ns}$ & $\mathrm{~ns}$ & $0.222^{*}$ & $0.212^{*}$ \\
321 & Lactobacillus & $*$ & 0.02 & 0.00 & $\mathrm{~ns}$ & $\mathrm{~ns}$ & $0.213^{*}$ & $\mathrm{~ns}$ \\
9 & Clostridiales & $* *$ & 1.88 & 1.33 & $\mathrm{~ns}$ & $\mathrm{~ns}$ & $0.207^{*}$ & $0.270^{* *}$ \\
203 & Clostridiales & $*$ & 0.03 & 0.10 & $\mathrm{~ns}$ & $\mathrm{~ns}$ & $-0.226^{*}$ & $-0.249^{*}$ \\
724 & Lactobacillus & $*$ & 0.00 & 0.03 & $\mathrm{~ns}$ & $\mathrm{~ns}$ & $-0.245^{*}$ & $\mathrm{~ns}$ \\
298 & Lachnospiraceae & $*$ & 0.21 & 0.09 & $\mathrm{~ns}$ & $\mathrm{~ns}$ & $\mathrm{~ns}$ & $0.214^{*}$ \\
14 & Ruminococcaceae & $* *$ & 0.04 & 0.28 & $\mathrm{~ns}$ & $\mathrm{~ns}$ & $\mathrm{~ns}$ & $-0.226^{*}$ \\
398 & Tenericutes order & $*$ & 0.02 & 0.12 & $\mathrm{~ns}$ & $\mathrm{~ns}$ & $\mathrm{~ns}$ & $-0.239^{*}$ \\
110 & Catabacteriaceae & $*$ & 0.03 & 0.13 & $\mathrm{~ns}$ & $\mathrm{~ns}$ & $\mathrm{~ns}$ & $-0.255^{*}$ \\
\hline
\end{tabular}

\section{Discussion}

259

260

261

262

263

264

265

266

267

268

269

270

271

272

273

274

275

276

277

278

279

\section{Between-diet comparisons of growth performance}

Overall, our results indicate that a sorghum-based diet increased AME, BWG and FE, but resulted in poorer FCR of chickens, compared to a wheat-based diet. These findings partially agree with other studies, showing that birds fed on a sorghum-based diet were more efficient in extracting energy from food (high AME values) (Black et al. 2005), and had higher BWG (Liu et al. 2015a; Selle et al. 2010; Torok et al. 2011) than those fed with wheat. However, contrary to our results, the latter studies found that sorghum-fed chickens had either better (Liu et al. 2015a; Selle et al. 2010; Torok et al. 2011) or similar feed conversion efficiency (Black et al. 2005) than wheat-fed chickens. Caecal microbiota produce short chain fatty acids which provide up to 10\% of energy to the bird and additionally, caecal contents backflow seed the gut, both up and downstream, via reverse peristalsis or reflux of digesta (Sonnenburg and Backhed 2016). These offer ways in which caecal microbiota are translocated into the intestinal tract thus affecting energy metabolism and performance both directly and indirectly.

Previous research indicated that chickens showing high AME values, tend to show low FCR values because of a low consumption of feed (Stanley et al. 2016). However, in our study, birds offered a sorghum-based diet ate more feed compared with those offered a wheat-based diet. These results, may suggest a preference for sorghum- vs. wheat-based diets in chickens, in agreement with Liu et al. (2015). Interestingly, only birds fed with a wheat-based diet showed a significant positive correlation between FE and FCR values (i.e. high feed intake leading to poor growth performance, or vice-versa). In either one or the other case, this correlation indicates that birds fed with wheat showed a trade-off between feed consumption and the ability to transform 
280 feed into body weight. Such trade-off did not occur in sorghum-fed chickens, suggesting that

281 their efficiency in converting feed to body mass was not compromised by the amount of feed

282 they ate. Despite this advantage, birds fed on a sorghum-based diet showed comparatively higher

283 FCR values than those fed on a wheat-based diet, indicating that energy in sorghum was used

284 less efficiently than the energy in wheat. The issue of sorghum-based diets in the Australian

285 poultry industry has been extensively reviewed (see Liu et al. [2015b] and references within),

286 and some studies have linked sorghum-based diets to inconsistent or suboptimal broiler

287 performance. These associations might have been produced by anti-nutritive factors present in

288 sorghum (Selle et al. 2013). Particularly, kafirin concentrations in Australian sorghum cultivars

289 might have increased as an accidental consequence of breeding programs. Also, these cultivars

290 contain phenolic compounds (Liu et al. 2015b). These factors could have contributed to the

291 lower utilisation of energy from sorghum-based diet relative to that from wheat-based diets

292 found in this study. Despite this potential disadvantage, Liu et al. (2015b) encouraged to rectify

293 these issues by using white sorghums with low kafirin levels. Our results support these findings

294 based on the positive effects that this diet had on AME and BWG values.

295 A number of studies have suggested a range of supplements can be added to sorghum-based diets

296 in order to improve productivity. For instance, reductions in FCR performance of cereal-fed

297 chickens could be improved by adding distillers dried grains with solubles (DDGS) (Jacobs \&

298 Parsons 2013). Also, the latter study indicated that DDGS might, under certain conditions, have

299 additional benefits by increasing AME, caecal microbiota, and gizzard size. Alternatively, Selle

300 et al. (2016) showed that adding sodium metabisulphite and protease in sorghum-based diets

301 enhanced starch utilisation, ultimately improving bird growth performance by decreasing FCR

302 and increasing AME and BWG. These supplements could improve the feed conversion

303 efficiency in sorghum-fed chickens.

304

305

306

307

308

309

\section{Diet-induced changes in caecal microbiota and associations with growth performance}

Diet has been recognised as a major modulator of intestinal microbiota. This conclusion has been mainly drawn from microbiota comparisons following the application of diets that are fundamentally very different, such as high fat vs. low fat or, even with more extreme differences, in plant based vs. Western diet (Sonnenburg and Backhed 2016). In these cases the differences in microbiota are just as extreme as the differences between the diets. Here we compared two 
310 similar grain-based diets and although we did find some differences, they were not large as the

311 differences in dietary compositions were not large either. Between-diet comparisons at the

312 microbiota community level showed differences in composition, but not in diversity. Differences

313 in composition were only detected when using Unweighted Unifrac Distance (presence-absence).

314 These results suggest that differences in diet produced a subtle yet significant impact in the

315 structure of the caecal microbial community, confirmed by between-diet shifts in abundance of

316 less than $19 \%$ of all OTUs. This is in contrast with Torok et al. (2011), who reported significant

317 differences in microbiota composition in the ileum, but not in the caecum of wheat-fed chickens

318 and sorghum-fed chickens. Despite the low effect of diet on caecal microbiota composition

319 found in our study, a number of caecal bacteria showed strong and contrasting correlations with

320 the growth performance of the birds. Particularly, our results show that the abundance of 30

321 OTUs were correlated with AME values. These results were supported by the finding that, at the

322 community level, microbiota composition was associated with AME values. Our results are

323 consistent with previous studies, since Torok et al. (2011) and Stanley et al. (2012) found

324 associations between the composition of caecal microbiota and avian growth performance, at

325 least in wheat-based diets.

326 Most of the microbiota in both dietary treatments belonged to the orders Lactobacillales and

327 Clostridiales, in agreement with other studies (Torok et al. 2011). Particularly, high numbers of

328 phylotypes assigned to Lactobacillus crispatus were recorded, yet these were much higher in

329 sorghum-fed birds than in wheat-fed birds, indicating that the abundance of this species is partly

330 influenced by diet-related differences. However, a study found that chickens fed with sorghum

331 showed lower numbers of lactobacilli than chickens fed with wheat (Shakouri et al. 2009), in

332 disagreement with our results. Generally, L. crispatus is considered beneficial because it

333 produces hydrogen peroxide, tolerates gastric juice and bile salts, and has high surface

334 hydrophobicity (Mota et al. 2006). This taxon, along with other strains, is involved in the

335 reduction of pathogen loads, such as Salmonella species (van der Wielen et al. 2002). Despite it

336 being generally recognised as a beneficial bacterium, its associations with chicken performance

337 have been mixed. Stanley et al. (2016) found that the abundance of L. crispatus was associated

338 with high bird performance, since a number of OTUs assigned to this taxon showed either

339 positive correlations with AME values or negative correlations with FCR values. Moreover,

340 Mignon-Grasteau et al. (2015) indicated that L. crispatus was associated with growth 
341 performance, since they found significantly lower abundance in high-FCR than in low-FCR

342 birds. Contrary to the latter two studies, bacterial phylotypes related to L. crispatus along with

343 two other Lactobacillus species, were linked to low avian growth performance (Torok et al.

344 2011). Also, Konsak et al. (2013) found that an OTU which was most closely related to $L$.

345 crispatus, was more abundant in birds with low AME values. Nevertheless, our results reinforce

346 those previously found by our group (Stanley et al. 2016), suggesting that the abundance of $L$.

347 crispatus is an indicator of high productivity, and that certain strains of this species may enhance

348 bird growth performance. Although the mechanisms behind this beneficial effect remain

349 unexplained, we hypothesise that L. crispatus could increase energy availability through

350 production of short-chained fatty acids. Our hypothesis is supported by Meimandipour et al.

351 (2010), who showed the butyrogenic effects of lactobacilli strains in a simulated chicken

352 caecum. These findings support the conclusion that some strains/OTUs most closely related to $L$.

353 crispatus could be performance-related bacteria that could be studied as candidates to enhance

354 productivity in poultry.

355 Additionally, sorghum-fed birds showed high abundance of another Lactobacillales member, the

356 Enterococcus genus. This genus encompasses several species, with a few considered beneficial,

357 such as E. faecium and E. faecalis (Devriese \& Pot 1995). Nevertheless, other species of

358 Enterococcus found in the gastro-intestinal tract of chickens have been associated with infectious

359 diseases, such as E. cecorum, related to osteomyelitis (Kense \& Landman 2011). One study

360 associated the increased abundance of the Enterococcus genus and the Enterobacteriaceae family

361 in the ileum with low feed conversion efficiency in sorghum-fed chickens (Lunedo et al. 2014).

362 In our study, we did not observe any significant correlations between Enterococcus and FCR, but

363 further research could clarify whether this genus is involved in the high FCR values observed in

364 sorghum-fed birds.

365 Several taxa within the order Clostridiales showed different abundances between dietary

366 treatments and contrasting correlations with growth performance measures. Particularly in this

367 group, the Lachnospiraceae family was the most abundant taxon, showing higher numbers in

368 sorghum- than in wheat-fed chickens. This family is a butyrate-producing group considered as

369 beneficial plant degraders and strong producers of short chain fatty acids (Biddle et al. 2013),

370 and its numbers are reduced in gastrointestinal illnesses such as chronic liver cirrhosis (Chen et

371 al. 2011) and inflammatory bowel disease (Berry \& Reinisch 2013). Lachnospiraceae have been 
372 associated with improved growth performance in chickens (low FCR values) (Stanley et al.

373 2012). Furthermore, Torok et al. (2011) found 4 OTUs assigned to Lachnospiraceae associated

374 with high performance (low FCR values), and 1 OTU assigned to the same taxon, associated

375 with low performance (high FCR values) in the caecal microbiota of chickens. Our results

376 support the latter two studies, since one OTU assigned to this family was found to be positively

377 correlated with BWG. Additionally, the Coprococcus genus was relatively more abundant in

378 sorghum- than in wheat-fed chickens. One OTU assigned to this genus was positively correlated

379 with BWG and FE, but another OTU was negatively correlated with FCR and AME, indicating

380 mixed associations with growth performance. Previously, Stanley et al. (2016) found three

381 different OTUs assigned to Coprococcus to be positively correlated with FCR, which indicates

382 that this genus might have negative consequences for chicken growth performance.

383 Also, there were three bacterial taxa within the Clostridiales order showing higher abundance in 384 wheat-fed than in sorghum-fed chickens: the families Catabacteriaceae and Clostridiales family 385 XIII, and the Clostridium leptum phylotype. Catabacteriaceae, a relatively unknown family that 386 has been found in polluted water (Codony et al. 2009), has been associated with poor feed 387 conversion efficiency in chickens (Stanley et al. 2016). Also, although little is known about 388 Clostridiales Family XIII, Singh et al. (2012) found Clostridiales Family XIII linked to broilers 389 showing high FCR values. Additionally, Steelman et al. (2012) found significantly higher 390 abundance of this taxon in horses with chronic laminitis than in healthy horses. In our study, we did not find any association between the three above-mentioned Clostridiales bacteria and chicken productivity. However, based on what other studies have reported, high abundance of these bacteria may result in poor bird performance. Future research efforts could study how differences in the proportions of cereals in feed influence the survival of these Clostridiales taxa in the chicken gut microbiota. Interestingly, a number of other bacteria within the Clostridiales order showed mixed associations with growth performance measures. Out of several OTUs (identified as either Clostridiales or Clostridium) which were prevalent in wheat-fed chickens, some showed negative correlations with AME, whereas others showed negative correlations with FCR. Such inconsistency of patterns might have been produced because Clostridiales is a high polyphyletic order. There seems to be some ambiguity in the taxonomy of several Clostridium species (Yutin \& Galperin 2013) despite Biddle et al. (2013) recommending major revisions. Species within this genus are a mixture of gram positive and negative bacteria, and some are not 
403

404

405

406

407

408

409

410

411

412

413

414

415

416

417

418

419

420

421

422

423

424

425

426

427

428

429

430

even anaerobes (Fåk \& Bäckhed 2012). Our results, in accordance with Stanley et al. (2016), suggest that some members of the Clostridium genus (from the Lachnospiraceae family) could be associated with better performance, whereas a number of other unidentified taxa in this order could be associated with low performance. Thus, in agreement with other studies (Rinttilä \& Apajalahti 2013; Stanley et al. 2016), the term clostridia should not be related with poor performance and/or health in chickens and beneficial and detrimental clostridia should be clearly distinguished.

\section{Conclusions}

In summary, our results suggest that differences in growth performance depending on cereal type used in the diet were correlated with changes in specific members of the microbiota in the caecum of chickens. Also, our study reinforces the hypothesis that specific bacteria could be used as probiotics to improve performance, given the associations of the caecal microbiota and growth performance measures that we found. Future studies could test this hypothesis in order to promote long-term sustainability for the poultry industry.

\section{Acknowledgements}

The data was analysed using the Isaac Newton High Performance Computing System at Central Queensland University. We wish to acknowledge the support from Jason Bell in all aspects of high performance computing. We also thank Derek Schultz, Evelyn Daniels and Kylee Swanson (SARDI) for their assistance with animal trials, and Honglei Chen (CSIRO) for operating the Roche 454 sequencer.

\section{References}

Anderson MJ. 2001. A new method for non-parametric multivariate analysis of variance. Austral Ecology 26:32-46.

Anderson MJ. 2006. Distance-based tests for homogeneity of multivariate dispersions. Biometrics 62:245-253.

Ashelford KE, Chuzhanova NA, Fry JC, Jones AJ, and Weightman AJ. 2005. At least 1 in 20 16S rRNA sequence records currently held in public repositories is estimated to contain substantial anomalies. Applied and Environmental Microbiology 71:7724-7736. 
431 Berry D, and Reinisch W. 2013. Intestinal microbiota: a source of novel biomarkers in

432

433

434

435

436

437

438

439

440

441

442

443

444

445

446

447

448

449

450

451

452

453

454

455

456

457

458

459 inflammatory bowel diseases? Best practice \& research Clinical gastroenterology 27:4758.

Biddle A, Stewart L, Blanchard J, and Leschine S. 2013. Untangling the genetic basis of fibrolytic specialization by Lachnospiraceae and Ruminococcaceae in diverse gut communities. Diversity 5:627-640.

Black J, Hughes R, Nielsen S, Tredrea A, MacAlpine R, and Van Barneveld R. 2005. The energy value of cereal grains, particularly wheat and sorghum, for poultry. Proceedings of the Australian Poultry Science Symposium 17:21-29.

Bragg L, Stone G, Imelfort M, Hugenholtz P, and Tyson GW. 2012. Fast, accurate errorcorrection of amplicon pyrosequences using Acacia. Nature methods 9:425-426.

Caporaso JG, Kuczynski J, Stombaugh J, Bittinger K, Bushman FD, Costello EK, Fierer N, Pena AG, Goodrich JK, and Gordon JI. 2010. QIIME allows analysis of high-throughput community sequencing data. Nature methods 7:335-336.

Chen Y, Yang F, Lu H, Wang B, Chen Y, Lei D, Wang Y, Zhu B, and Li L. 2011. Characterization of fecal microbial communities in patients with liver cirrhosis. Hepatology 54:562-572.

Choct M, and Hughes R. 1999. Chemical and physical characteristics of grains related to variability in energy and amino acid availability in poultry. Crop and Pasture Science 50:689-702.

Choct M, Hughes R, and Annison G. 1999. Apparent metabolisable energy and chemical composition of Australian wheat in relation to environmental factors. Australian Journal of Agricultural Research 50:447-451.

Chun J, Lee J-H, Jung Y, Kim M, Kim S, Kim BK, and Lim Y-W. 2007. EzTaxon: a web-based tool for the identification of prokaryotes based on 16S ribosomal RNA gene sequences. International journal of systematic and evolutionary microbiology 57:2259-2261.

Codony F, Adrados B, Pérez LM, Fittipaldi M, and Morató J. 2009. Detection of Catabacter hongkongensis in polluted European water samples. Journal of Zhejiang University Science B 10:867-869. 
460

461

462

463

464

465

466

467

468

469

470

471

472

473

474

475

476

477

478

479

480

481

482

483

484

485

486

487

488

DeSantis TZ, Hugenholtz P, Larsen N, Rojas M, Brodie EL, Keller K, Huber T, Dalevi D, Hu P, and Andersen GL. 2006. Greengenes, a chimera-checked 16S rRNA gene database and workbench compatible with ARB. Applied and Environmental Microbiology 72:50695072.

Devriese L, and Pot B. 1995. The genus Enterococcus. In: Wood BJB, and Holzapfel WH, eds. The genera of lactic acid bacteria. New York: Springer, 327-367.

Edgar RC. 2010. Search and clustering orders of magnitude faster than BLAST. Bioinformatics 26:2460-2461.

Fåk F, and Bäckhed F. 2012. Lactobacillus reuteri prevents diet-induced obesity, but not atherosclerosis, in a strain dependent fashion in Apoe-/- mice. PloS one 7:e46837.

Hübener K, Vahjen W, and Simon O. 2002. Bacterial responses to different dietary cereal types and xylanase supplementation in the intestine of broiler chicken. Archives of Animal Nutrition 56:167-187.

Jacobs C, and Parsons CM. 2013. The effects of coarse ground corn, whole sorghum, and a prebiotic on growth performance, nutrient digestibility, and cecal microbial populations in broilers fed diets with and without corn distillers dried grains with solubles. Poultry Science 92:2347-2357.

Kense M, and Landman WJ. 2011. Enterococcus cecorum infections in broiler breeders and their offspring: molecular epidemiology. Avian pathology 40:603-612.

Konsak BM, Stanley D, Haring VR, Geier MS, Hughes RJ, Howarth GS, Crowley TM, and Moore RJ. 2013. Identification of differential duodenal gene expression levels and microbiota abundance correlated with differences in energy utilisation in chickens. Animal Production Science 53:1269-1275.

Lane D. 1991. 16S/23S rRNA sequencing. In: Stackebrandt E, and Goodfellow M eds. Nucleic acid techniques in bacterial systematics. New York: John Wiley and Sons, 115-175.

Liu S, Cadogan D, Péron A, Truong H, and Selle P. 2014. Effects of phytase supplementation on growth performance, nutrient utilization and digestive dynamics of starch and protein in broiler chickens offered maize-, sorghum- and wheat-based diets. Animal Feed Science and Technology 197:164-175. 
489 490

491

492

493

494

495

496

497

498

499

500

501

502

503

504

505

506

507

508

509

510

511

512

513

514

515

516

517

Liu S, Cadogan D, Péron A, Truong H, and Selle P. 2015a. A combination of xylanase, amylase and protease influences growth performance, nutrient utilisation, starch and protein digestive dynamics in broiler chickens offered maize-, sorghum-and wheat-based diets. Animal Production Science 55:1255-1263.

Liu SY, Fox G, Khoddami A, Neilson KA, Truong HH, Moss AF, and Selle PH. 2015b. Grain sorghum: a conundrum for chicken-meat production. Agriculture 5:1224-1251.

Liu SY, Selle PH, and Cowieson AJ. 2013. Strategies to enhance the performance of pigs and poultry on sorghum-based diets. Animal Feed Science and Technology 181:1-14.

Lozupone C, and Knight R. 2005. UniFrac: a new phylogenetic method for comparing microbial communities. Applied and Environmental Microbiology 71:8228-8235.

Lunedo R, Fernandez-Alarcon M, Carvalho F, Furlan L, and Macari M. 2014. Analysis of the intestinal bacterial microbiota in maize-or sorghum-fed broiler chickens using real-time PCR. British poultry science 55:795-803.

Meimandipour A, Shuhaimi M, Soleimani AF, Azhar K, Hair-Bejo M, Kabeir BM, Javanmard A, Anas OM and Yazid AM. 2010. Selected microbial groups and short-chain fatty acids profile in a simulated chicken cecum supplemented with two strains of Lactobacillus. Poultry science 89:470-476.

Mignon-Grasteau S, Narcy A, Rideau N, Chantry-Darmon C, Boscher M-Y, Sellier N, Chabault M, Konsak-Ilievski B, Le Bihan-Duval E, and Gabriel I. 2015. Impact of selection for digestive efficiency on microbiota composition in the chicken. PloS one 10:e0135488.

Mota RM, Moreira JLS, Souza MR, Horta MF, Teixeira SM, Neumann E, Nicoli JR, and Nunes ÁC. 2006. Genetic transformation of novel isolates of chicken Lactobacillus bearing probiotic features for expression of heterologous proteins: a tool to develop live oral vaccines. BMC biotechnology 6:1.

Quinlan AR, Stewart DA, Strömberg MP, and Marth GT. 2008. Pyrobayes: an improved base caller for SNP discovery in pyrosequences. Nature methods 5:179-181.

Rehman HU, Vahjen W, Awad WA, and Zentek J. 2007. Indigenous bacteria and bacterial metabolic products in the gastrointestinal tract of broiler chickens. Archives of Animal Nutrition 61:319-335. 
518 Rinttilä T, and Apajalahti J. 2013. Intestinal microbiota and metabolites-Implications for 519 broiler chicken health and performance1. The Journal of Applied Poultry Research $520 \quad 22: 647-658$.

521 Round JL, and Mazmanian SK. 2009. The gut microbiota shapes intestinal immune responses 522 during health and disease. Nature Reviews Immunology 9:313-323.

523 Selle P, Cadogan D, Ru Y, and Partridge G. 2010. Impact of exogenous enzymes in sorghum-or 524 wheat-based broiler diets on nutrient utilization and growth performance. International Journal of Poultry Science 9:53-58.

526 Selle P, Liu S, and Cowieson A. 2013. Sorghum: An enigmatic grain for chicken-meat production. In: Parra PC, ed. Sorghum: production, growth habits and health benefits. Hauppauge: Nova Publishers Inc, 1-44.

529 Selle PH, Truong HH, McQuade LR, Moss AF, and Liu SY. 2016. Reducing agent and 530 exogenous protease additions, individually and in combination, to wheat- and sorghumbased diets interactively influence parameters of nutrient utilisation and digestive

Shakouri M, Iji P, Mikkelsen L, and Cowieson A. 2009. Intestinal function and gut microflora of 535

broiler chickens as influenced by cereal grains and microbial enzyme supplementation. dynamics in broiler chickens. Animal Nutrition.

http://dx.doi.org/10.1016/j.aninu.2016.08.001 Journal of animal physiology and animal nutrition 93:647-658.

Singh K, Shah T, Deshpande S, Jakhesara S, Koringa P, Rank D, and Joshi C. 2012. High through put 16S rRNA gene-based pyrosequencing analysis of the fecal microbiota of high FCR and low FCR broiler growers. Molecular biology reports 39:10595-10602.

Snell-Castro R, Godon J-J, Delgenès J-P, and Dabert P. 2005. Characterisation of the microbial diversity in a pig manure storage pit using small subunit rDNA sequence analysis. FEMS

543 Sonnenburg JL, Backhed F. 2016. Diet-microbiota interactions as moderators of human 544 metabolism. Nature 535:56-64. 
545 Stanley D, Denman SE, Hughes RJ, Geier MS, Crowley TM, Chen H, Haring VR, and Moore

546 RJ. 2012. Intestinal microbiota associated with differential feed conversion efficiency in 547 chickens. Applied microbiology and biotechnology 96:1361-1369.

548 Stanley D, Geier MS, Denman SE, Haring VR, Crowley TM, Hughes RJ, and Moore RJ. 2013.

549 Identification of chicken intestinal microbiota correlated with the efficiency of energy 550 extraction from feed. Veterinary microbiology 164:85-92.

551 Stanley D, Hughes RJ, Geier MS, and Moore RJ. 2016. Bacteria within the gastrointestinal tract

552

553

554

555

556

557

558

559

560

561

562 563

564

565

566

567

568

569

570

571

572 microbiota correlated with improved growth and feed conversion: challenges presented for the identification of performance enhancing probiotic bacteria. Front Microbiol $7: 187$.

Steelman SM, Chowdhary BP, Dowd S, Suchodolski J, and Janečka JE. 2012. Pyrosequencing of 16S rRNA genes in fecal samples reveals high diversity of hindgut microflora in horses and potential links to chronic laminitis. BMC veterinary research 8:1.

Torok VA, Hughes RJ, Mikkelsen LL, Perez-Maldonado R, Balding K, MacAlpine R, Percy NJ, and Ophel-Keller K. 2011. Identification and characterization of potential performancerelated gut microbiotas in broiler chickens across various feeding trials. Applied and Environmental Microbiology 77:5868-5878.

Tremaroli V, and Bäckhed F. 2012. Functional interactions between the gut microbiota and host metabolism. Nature 489:242-249.

van der Wielen PW, Lipman LJ, van Knapen F, and Biesterveld S. 2002. Competitive exclusion of Salmonella enterica serovar Enteritidis by Lactobacillus crispatus and Clostridium lactatifermentans in a sequencing fed-batch culture. Applied and Environmental Microbiology 68:555-559.

$\mathrm{Yu}$ Z, and Morrison M. 2004. Improved extraction of PCR-quality community DNA from digesta and fecal samples. Biotechniques 36:808-813.

Yutin N, and Galperin MY. 2013. A genomic update on clostridial phylogeny: Gram-negative spore formers and other misplaced clostridia. Environmental microbiology 15:2631-2641. 